\title{
Solution of Second Order Singular Perturbed Delay Differential Equation Using Trigonometric B-Spline
}

\author{
Mandeep Kaur Vaid, Geeta Arora* \\ Department of Mathematics \\ Lovely Professional University, Phagwara, Punjab, India \\ Corresponding author: geetadma@gmail.com
}

(Received August 31, 2018; Accepted November 21, 2018)

\begin{abstract}
In this paper, a numerical technique is presented to approximate the solution of a singular perturbed delay differential equation. The continual emerge of singular perturbed delay differential equations in a mathematical model of real life applications trigger the researchers for the numerical treatment of these equations. The numerical technique is based on trigonometric cubic B-spline functions in which derivatives are approximated as a linear sum of basis functions. The obtained matrix system is solved by using the Thomas Algorithm. The convergence of the employed proposal is scrutinized and computational work is carried out on four examples to test the capability of the proposed scheme. The approximated solution is compared with the existing technique and to present the behavior of the obtained solution graphs are plotted.
\end{abstract}

Keywords-Perturbed, Delay, B-spline, Collocation method, Truncation error.

\section{Introduction}

Mathematical modeling of numerous real life phenomenon results in ordinary differential equations which help the researchers to solve environmental issues such as pollution, cyber security and so on. One such model that discussed the water pollutants and outturn as a system of nonlinear ordinary differential equation is given by Shah et al. (2018). Another mathematical model narrating vulnerabilities is proposed by Bhatt et al. (2017). The objective of this model is to capture the supplementary bugs in programming during invention processes. One of the mathematical models proposed by Deepika et al. (2017) depicts the growth model related to the reliability concept.

Mathematical subject involving the study of singular perturbed delay differential equations (SPDDE) has become a full-fledged research with a long background. These equations are prominent for their extended implementations in every part of science and engineering. These differential equations are ordinary differential equations and embrace two small parameters, known as the perturbation parameter and delay parameter. The perturbation parameter $\varepsilon$ is multiplied with higher order derivative term of SPDDE and arises due to the clampdown in the small parameter of the physical systems which in turns reduce the order of system as $\varepsilon \rightarrow 0$. For instance, in control theory small parameters such as moments of inertia, resistances and so on when subdued reduce the order of the system. And another small parameter, delay parameter exists in the argument of the reaction term which appears due to the participation of feedback which is obligatory in order to avoid a rickety state. But the feedback control demand a definite time to sense information and to react on it. Hence, the formulation of a delay differential equation is a conventional way to elucidate these processes. 
International Journal of Mathematical, Engineering and Management Sciences

Vol. 4, No. 2, 349-360, 2019

https://dx.doi.org/10.33889/IJMEMS.2019.4.2-028

The flourish concern in the numerical study of SPDDE is due to their wide range of applications. These differential equations arise in mathematical modeling of many real life applications for instant, in biosciences (Rihan, 2013), in control theory (Stein, 1965; Stein, 1967), in study of human pupil reflex (Longtin and Milton, 1988), physical and biological phenomena like optically bistable devices (Derstine et al., 1982; Bocharov and Rihan, 2000), in study of HIV-1 infection (Nelson and Perelson, 2002), physical control systems (Mackey and Glass, 1977), signal transmission (Lange and Miura, 1982) etc.

The numerical solution of SPDDE has given by many researchers by using various numerical techniques. Some of these methods can be summarized as: File et al. (2017) presented a numerical method to find the solution of SPDDE by using fourth order finite difference method. A novel method based on Numerov's method elucidated by Chakravarthy and Kumar (2017) for the numerical solution of singular perturbed delay of reaction diffusion type. Sirisha and Reddy (2017) proposed a numerical integration technique with an exponential integration factor for the numerical solution of SPDDE. Mahendran and Subburayan (2018) extended a fitted finite difference method for third order singular perturbed delay of convention diffusion type. Swamy et al. (2015) presented a computational method for SPDDE in 2014. Recently, Kanth and Kumar (2018) proposed a parametric spline scheme for a class of nonlinear SPDDE.

With this motivation, trigonometric cubic B-spline collocation method is used to solve singular perturbed delay differential equations. The present scheme is used to approximate the solution of the equation by fitting a mesh to capture the conduct of the solution. In section 2 , the considered problem is described. The discussion of the used numerical scheme is accomplished in section 3 . In section 4, the convergence analysis of the proposed scheme is recounted followed by numerical computations executed on 4 examples to test the efficiency of the scheme in section 5. Section 6 is embracing conclusions with the discussion.

\section{Description of Problem}

Consider the singularly perturbed delay differential equation of the form

$L y \equiv \varepsilon y^{\prime \prime}(x)+a(x) y(x-\delta)+b(x) y(x)=f(x), 0<x<1$

subject to the boundary conditions,

$y(x)=\phi(x), x \in[-\delta, 0]$ and $y(1)=\gamma$

where $0<\varepsilon<<1$ and $\phi(\mathrm{x})$ is sufficiently smooth on $[-1,0]$ and $\beta$ is a given constant which is independent of $\varepsilon$.

Taylor's series is used to handle the delay term given by

$$
y(x-\delta)=y(x)-\delta y^{\prime}(x) \text {. }
$$

On substituting above delay term relation in equation (1), we get the equation

$\varepsilon y^{\prime \prime}(x)-a(x) \delta y^{\prime}(x)+(a(x)+b(x)) y(x)=f(x)$

Re-writing above equation we get

$p(x) y^{\prime \prime}(x)+y^{\prime}(x)+s(x) y(x)=f(x)$

where $\mathrm{p}(\mathrm{x})=\varepsilon, \mathrm{q}(\mathrm{x})=-\mathrm{b}(\mathrm{x}) \delta$ and $\mathrm{s}(\mathrm{x})=a(x)+b(x)$. 
International Journal of Mathematical, Engineering and Management Sciences

Vol. 4, No. 2, 349-360, 2019

https://dx.doi.org/10.33889/IJMEMS.2019.4.2-028

\section{Trigonometric Cubic B-Spline Collocation Method}

The numerical solution of equation (1)-(2) is obtained by using trigonometric cubic B-spline collocation method. The set of functions $\left\{T_{-1}, T_{0}, T_{1}, \ldots \ldots \ldots, T_{N}, T_{N+1}\right\}$, form a basis for function defined over the region $0 \leq x \leq 1$. The domain given by $0 \leq x \leq 1$ for the considered problem is partitioned into a uniform mesh $\left\{0=x_{0}<x_{1}<x_{2}<\cdots \ldots<x_{N}=l\right\}$. The length of each sub intervals is given by $\mathrm{h}$ where $\mathrm{h}=x_{i+1}-x_{i}, \mathrm{i}=0,1,2, \ldots \mathrm{N}-1$. An approximation to the solution can be expressed in terms of trigonometric B-splines as

$\mathrm{y}(\mathrm{x})=\sum_{i=-1}^{N+1} \alpha_{i} T_{i}(x)$

where $\alpha_{i}{ }^{\prime} s$ are unknown real coefficients to be decided with the constraint that $\mathrm{y}(\mathrm{x})$ satisfies the considered problem at $\mathrm{N}+1$ collocation points as well at the boundary conditions and $T_{i}(x)^{\prime} s$ are the Trigonometric B-spline basis functions. For the trigonometric cubic B-spline function $T_{i}$ and for the values of $T_{i}(x), T_{i}{ }^{\prime}(x), T_{i}{ }^{\prime \prime}(x)$ at nodal points refer Dag et al. (2014).

Now, by using trigonometric cubic B-spline basis functions and their values at the nodal points, the value of $y(x)$ and its first and second order derivative can be expressed in terms of $\alpha_{i}{ }^{\prime} s$ as

$$
\begin{gathered}
y\left(x_{i}\right)=k_{1} a_{i-1}+k_{2} a_{i}+k_{1} a_{i+1} \\
y^{\prime}\left(x_{i}\right)=k_{3} a_{i-1}+k_{4} a_{i+1} \\
y^{\prime \prime}\left(x_{i}\right)=k_{5} a_{i-1}+k_{6} a_{i}+k_{5} a_{i+1}
\end{gathered}
$$

where $k_{1}=\sin ^{2}\left(\frac{h}{2}\right) \csc (h) \csc \left(\frac{3 h}{2}\right), k_{2}=\frac{2}{1+2 \cos (h)}, k_{3}=-\frac{3}{4} \csc \left(\frac{3 h}{2}\right), k_{4}=\frac{3}{4} \csc \left(\frac{3 h}{2}\right)$, $k_{5}=\frac{3\left((1+3 \cos (h)) \cos ^{2}\left(\frac{h}{2}\right)\right)}{16\left(2 \cos \left(\frac{h}{2}\right)+\cos (3 h / 2)\right)}$ and $k_{6}=-\frac{3 \cot ^{2}\left(\frac{h}{2}\right)}{2+4 \cos (h)}$.

To apply the collocation technique, collocation points are selected in such a way that they concur with the nodal points. On substituting the values of $y_{i}, y_{i}^{\prime}$ and $y_{i}^{\prime \prime}$ at nodal points in equation (4) we get a system of $(\mathrm{N}+1)$ linear equations in $(\mathrm{N}+3)$ unspecified variables as

$E_{i}^{l} \alpha_{i-1}+E_{i} \alpha_{i}+E_{i}^{r} \alpha_{i+1}=f_{i}, \quad 0 \leq i \leq N$

where $E_{i}^{l}=p(x) k_{5}+q(x) k_{3}+s(x) k_{1}, E_{i}=p(x) k_{6}+s(x) k_{2}$ and $E_{i}^{r}=p(x) k_{5}+q(x) k_{4}+s(x) k_{1}$.

Now, in order to obtain a system of equations with number of variables equal to the number of equations we have used boundary conditions to calculate the value of two unknowns $\alpha_{-1}$ and $\alpha_{n+1}$.

When $i=0$ in equation (6) there exist an unspecified variable $\alpha_{-1}$ and similarly, for $i=n$ in equation (6) variable $\alpha_{n+1}$ exists. 
International Journal of Mathematical, Engineering and Management Sciences

Vol. 4, No. 2, 349-360, 2019

https://dx.doi.org/10.33889/IJMEMS.2019.4.2-028

From boundary conditions, we get $\alpha_{-1}=\frac{\emptyset_{0}-k_{2} \alpha_{0}-k_{1} \alpha_{1}}{k_{1}}$ and $\alpha_{n+1}=\frac{\gamma-k_{1} \alpha_{n-1}-k_{2} \alpha_{n}}{k_{1}}$.

By using the value of $\alpha_{-1}$ and $\alpha_{n+1}$ in the above system, we get

$\alpha_{0}\left(\frac{-k_{2}}{k_{1}} E_{0}^{l}+E_{0}\right)+\alpha_{1}\left(E_{0}^{r}-E_{0}^{l}\right)=f_{0}-\frac{\varnothing E_{0}{ }^{l}}{k_{1}}$

and

$\alpha_{n-1}\left(E_{n}{ }^{l}-E_{i}^{r}\right)+\alpha_{n}\left(E_{n}-\frac{k_{2}}{k_{1}} E_{n}^{r}\right)=f_{n}-\frac{\gamma E_{n}^{r}}{k_{1}}$

Now we have $(\mathrm{N}+1)$ linear equations in $(\mathrm{N}+1)$ variables which can be expressed as matrix system: $\mathrm{AY}=\mathrm{B}$ where $\mathrm{Y}=\alpha_{0}, \alpha_{1}, \alpha_{2}, \ldots \ldots \ldots, \alpha_{N}$.

The coefficient matrix $\mathrm{A}$ is given by

$\mathrm{A}=\left[\begin{array}{cccccccc}E_{0} & E_{0}^{r} & \ldots & \ldots & \cdots & \ldots & \ldots & 0 \\ E_{1}^{l} & E_{1} & E_{1}^{r} & \ldots & \ldots & \ldots & \ldots & 0 \\ \vdots & \vdots & \vdots & \vdots & \vdots & \vdots & \vdots & \vdots \\ \vdots & \vdots & \vdots & \vdots & \vdots & \vdots & \vdots & \vdots \\ 0 & \cdots & 0 & E_{i}^{l} & E_{i} & E_{i}^{r} & 0 & \ldots \\ \vdots & \vdots & \vdots & \vdots & \vdots & \vdots & \vdots & \vdots \\ \vdots & \vdots & \vdots & \vdots & \vdots & \vdots & \vdots & \vdots \\ 0 & \cdots & \ldots & \ldots & \cdots & E_{n-1}^{l} & E_{n-1} & E_{n-1}^{r} \\ 0 & \ldots & \ldots & \ldots & \ldots & \cdots & E_{n} & E_{n}^{r}\end{array}\right] ; \mathrm{B}=\left[\begin{array}{c}f\left(x_{0}\right)-E_{0}^{l}\left(\frac{\phi_{0}}{k_{1}}\right) \\ f\left(x_{1}\right) \\ \vdots \\ \vdots \\ \vdots \\ f\left(x_{n-1}\right) \\ f\left(x_{n}\right)-\gamma\left(\frac{E_{n}^{r}}{k_{1}}\right)\end{array}\right]$

The matrix $\mathrm{A}$ is a tridiagonal dominant matrix such that $|A| \neq 0$. Hence, the matrix $\mathrm{A}$ is non singular and the solution of matrix system $\mathrm{A} \alpha=\mathrm{B}$ can be calculated to find the values of all $\alpha_{i}{ }^{\prime} s$ with the boundary conditions.

\section{Convergence Analysis}

In this section, we have discussed the convergence analysis of the method. With an assumption that the function $\mathrm{y}(\mathrm{x})$ is a function with continuous derivatives over the entire domain $[0,1]$.The tri-diagonal system of the matrix defined above can be written as,

$A Y=B$ where $A=\left(m_{i, j}\right), 0 \leq i, j \leq N$ is a tridiagonal matrix with

$m_{i, i-1}=E_{i}^{l}, m_{i, i}=E_{i}$ and $m_{i, i+1}=E_{i}^{r}$ for $\mathrm{i}=1,2,3,4, \ldots \ldots, \mathrm{N}-1$

$m_{0,0}=E_{0}, m_{0,1}=E_{0}^{r}, m_{N-1, N}=E_{N}$ and $m_{N, N}=E_{N}^{r}$ 
International Journal of Mathematical, Engineering and Management Sciences

Vol. 4, No. 2, 349-360, 2019

https://dx.doi.org/10.33889/IJMEMS.2019.4.2-028

with local truncation error

$$
T_{i}\left(h_{i}\right)=h^{2}\left[\left(\frac{k_{2}}{2\left(2 k_{1}+k_{2}\right)}\right)\left(\frac{k_{3}+k_{4}}{2 k_{1}+k_{2}}\right) y^{\prime}\right]+o\left(h^{3}\right)
$$

and $Y=\left(\mathrm{y}_{1}, \mathrm{y}_{2}, \mathrm{y}_{3}, \ldots, \mathrm{y}_{\mathrm{n}-1}\right)^{\mathrm{t}}$.

Also if $\bar{Y}=\left(\overline{y_{1}}, \overline{y_{2}}, \overline{y_{3}}, \ldots . \overline{y_{n-1}}\right)^{t}$ is the exact solution and the local truncation error is given by $T(h)=\left(T_{1}\left(h_{1}\right), T_{2}\left(h_{2}\right), T_{3}\left(h_{3}\right), \ldots \ldots \ldots T_{n-1}\left(h_{n-1}\right)\right)^{t}$, then we have $A \bar{Y}-T(h)=C$, where $C$ is any constant.

Therefore, we have $A(\bar{Y}-Y)=T(h)$.

The error equation can be written as

$A E=T(h)$

where $E=\bar{Y}-Y=\left(e_{1}, e_{2}, e_{3}, \ldots \ldots \ldots e_{n-1}\right)^{t}$.

Hence from equation (9),

$E=A^{-1} T(h)$

The choice of $\mathrm{h}$ is made sufficiently small, so that matrix A is irreducible and monotone. Thus $A^{-1}$ exists.

From the theory of matrices it is known that $\sum_{i=0}^{n} \bar{m}_{k, i} S_{i}=1$, where $\bar{m}_{k, i}$ is $(\mathrm{k}, \mathrm{i})$ the element of the $A^{-1}$.

Here we have $S_{i}=\sum_{j=1}^{n-1} m_{i, j}=h^{0} B_{i}$,

$B_{i}=\left\{\begin{array}{c}p\left(x_{i}\right)\left(k_{5}+k_{6}\right)+q\left(x_{i}\right)\left(k_{4}\right)+s\left(x_{i}\right)\left(k_{1}+k_{2}\right) \text { for } i=0 \\ p\left(x_{i}\right)\left(2 k_{5}+k_{6}\right)+q\left(x_{i}\right)\left(k_{3}+k_{4}\right)+s\left(x_{i}\right)\left(2 k_{1}+k_{2}\right) \text { for } \mathrm{i}=1,2,3, \ldots, \mathrm{N}-1 . \\ p\left(x_{i}\right)\left(k_{5}+k_{6}\right)+q\left(x_{i}\right)\left(k_{4}\right)+s\left(x_{i}\right)\left(k_{1}+k_{2}\right) \text { for } i=N\end{array}\right.$

Therefore, $\sum_{i=0}^{n} m_{k, i} \leq \frac{1}{\min \left(S_{i}\right)} \leq \frac{1}{\left|B_{i}\right|}$.

Thus from the equation (10), the element wise error is $e_{j}=\sum_{i=0}^{n} m_{k, i} T_{i}(h)$, for $\mathrm{j}=0,1,2,3, \ldots \ldots, \mathrm{n}$ implies the result that $e_{j} \leq \frac{k h^{2}}{\left|B_{i}\right|}$ where $k$ is a constant independent of $h$.

Therefore, $\|E\|=O\left(h^{2}\right)$.

This concludes that our method is second order convergent for the uniform mesh. 
International Journal of Mathematical, Engineering and Management Sciences

Vol. 4, No. 2, 349-360, 2019

https://dx.doi.org/10.33889/IJMEMS.2019.4.2-028

\section{Numerical Examples}

We have obtained the numerical solution of some examples by the presented scheme in order to show the efficiency of the method. As the exact of the equations are not known hence the double mesh principle is used to calculate the maximum absolute error.

Example 1. Let us consider equation $\varepsilon y^{\prime \prime}(x)-2 y(x-\delta)-y(x)=1$ subject to the boundary conditions

$$
y(x)=1,-\delta \leq x \leq 0 \text { and } y(1)=0
$$

The considered equation is obtained from equation (1) for $a(x)=-2, b(x)=-1$ with $f(x)=1$. This example is illustrating the behavior of SPDDE with the left layer. The maximum absolute error obtained for this example for diverse values of delay parameter and for different values of the perturbed parameter are presented in Table 1 and Table 2 respectively. The results are compared with the existing method and it is found that results by our method are better as compared to the discussed method. The conduct of the solution is presented in Figure 1.

Table 1. The maximum error obtained for $\boldsymbol{\varepsilon}=0.1$ and for different values of $\mathrm{N}$ and $\boldsymbol{\delta}$ for Example 1

\begin{tabular}{|c|l|l|l|}
\hline$\delta$ & $\mathrm{N}=100$ & $\mathrm{~N}=200$ & $\mathrm{~N}=400$ \\
\hline 0.03 & $5.7000 \mathrm{e}-005$ & $5.0000 \mathrm{e}-006$ & $4.0000 \mathrm{e}-006$ \\
\hline 0.05 & $3.9000 \mathrm{e}-005$ & $2.5000 \mathrm{e}-005$ & $5.0000 \mathrm{e}-006$ \\
\hline 0.09 & $7.1000 \mathrm{e}-003$ & $1.4000 \mathrm{e}-005$ & $3.0000 \mathrm{e}-006$ \\
\hline Swamy et al. (2015) & \multicolumn{5}{l}{} \\
\hline 0.03 & $3.1674 \mathrm{e}-003$ & $1.6058 \mathrm{e}-003$ & $8.0837 \mathrm{e}-004$ \\
\hline 0.05 & $8.7514 \mathrm{e}-003$ & $4.7344 \mathrm{e}-003$ & $2.4561 \mathrm{e}-004$ \\
\hline 0.09 & $3.0784 \mathrm{e}-003$ & $1.5660 \mathrm{e}-003$ & $7.9000 \mathrm{e}-004$ \\
\hline
\end{tabular}

Table 2. The maximum absolute error of Example 1 for $\boldsymbol{\delta}=\mathbf{0 . 5} \boldsymbol{\varepsilon}$

\begin{tabular}{|c|c|c|c|c|}
\hline$\varepsilon$ & $N=2^{-4}$ & $N=2^{-5}$ & $N=2^{-6}$ & $N=2^{-7}$ \\
\hline $2^{-4}$ & $2.906 \mathrm{e}-003$ & $7.13 e-004$ & $1.82 \mathrm{e}-004$ & $4.00 \mathrm{e}-005$ \\
\hline $2^{-5}$ & $5.226 \mathrm{e}-003$ & $1.262 \mathrm{e}-003$ & $3.14 \mathrm{e}-004$ & $7.80 \mathrm{e}-005$ \\
\hline $2^{-6}$ & $1.071 \mathrm{e}-002$ & $2.485 \mathrm{e}-003$ & $6.110 \mathrm{e}-004$ & $1.520 \mathrm{e}-004$ \\
\hline \multicolumn{5}{|c|}{ Swamy et al. (2015) } \\
\hline $2^{-4}$ & $2.1118 \mathrm{e}-002$ & $1.1692 \mathrm{e}-002$ & $6.1941 \mathrm{e}-003$ & $3.1887 \mathrm{e}-003$ \\
\hline $2^{-5}$ & $2.7872 \mathrm{e}-002$ & $1.6023 \mathrm{e}-002$ & $8.6367 \mathrm{e}-003$ & $4.4957 \mathrm{e}-003$ \\
\hline $2^{-6}$ & $3.5711 \mathrm{e}-002$ & $2.1293 \mathrm{e}-002$ & $1.1869 \mathrm{e}-002$ & $6.2731 \mathrm{e}-003$ \\
\hline
\end{tabular}


International Journal of Mathematical, Engineering and Management Sciences

Vol. 4, No. 2, 349-360, 2019

https://dx.doi.org/10.33889/IJMEMS.2019.4.2-028

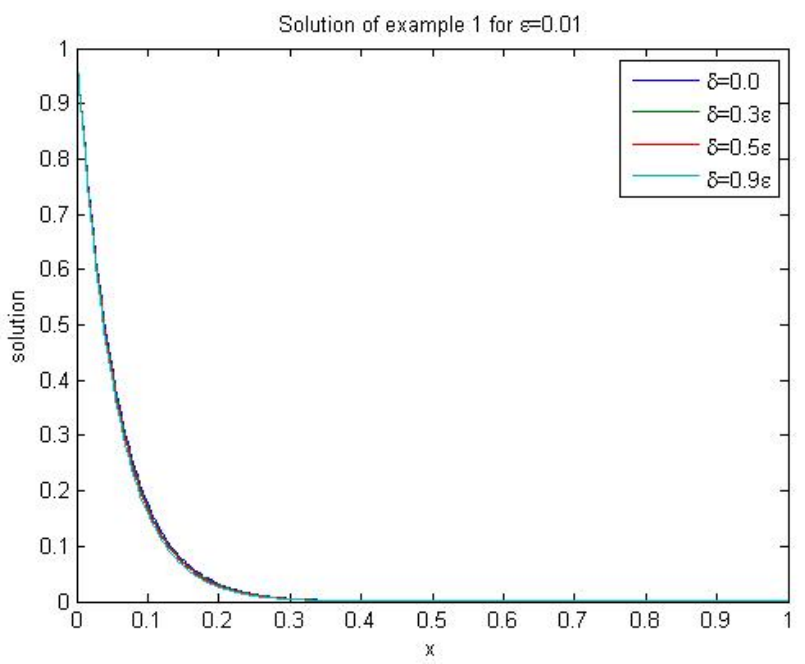

Figure 1. Numerical solution of Example 1 with different values of $\boldsymbol{\delta}$ with $\boldsymbol{\varepsilon}=0.01$

Example 2. Consider the equation $\varepsilon y^{\prime \prime}(x)+0.25 y(x-\delta)-y(x)=1$ with boundary conditions given by

$$
y(x)=1,-\delta \leq x \leq 0 \text { and } y(1)=0 .
$$

The above equation is obtained from equation (1) for $a(x)=0.25, b(x)=-1$ with $f(x)=1$. This example is a SPDDE with left layer and the behavior of the solution obtained for different values of delay parameters is shown in Figure 2. The maximum absolute error obtained for various values of the delay parameter and for different values of the perturbed parameter is presented in Table 3 and Table 4 respectively.

Table 3. The maximum error obtained for $\boldsymbol{\varepsilon}=0.1$ for different values of $\mathrm{N}$ and $\boldsymbol{\delta}$ for Example 2

\begin{tabular}{|c|c|c|c|}
\hline$\delta$ & $\mathrm{N}=100$ & $\mathrm{~N}=200$ & $\mathrm{~N}=400$ \\
\hline 0.03 & $3.3000 \mathrm{e}-005$ & $6.1000 \mathrm{e}-005$ & $9.0000 \mathrm{e}-006$ \\
\hline 0.05 & $2.5000 \mathrm{e}-005$ & $5.0000 \mathrm{e}-006$ & $1.8000 \mathrm{e}-005$ \\
\hline 0.09 & $6.0000 \mathrm{e}-006$ & $7.0000 \mathrm{e}-006$ & $2.1000 \mathrm{e}-005$ \\
\hline \multicolumn{5}{|l|}{} \\
\hline Swamy et al. (2015) & $2.1999 \mathrm{e}-003$ & $1.1041 \mathrm{e}-003$ & $5.5315 \mathrm{e}-004$ \\
\hline 0.03 & $2.2012 \mathrm{e}-003$ & $1.1049 \mathrm{e}-003$ & $5.5345 \mathrm{e}-004$ \\
\hline 0.05 & $2.1999 \mathrm{e}-003$ & $1.1038 \mathrm{e}-003$ & $5.5289 \mathrm{e}-004$ \\
\hline
\end{tabular}


International Journal of Mathematical, Engineering and Management Sciences

Vol. 4, No. 2, 349-360, 2019

https://dx.doi.org/10.33889/IJMEMS.2019.4.2-028

Table 4. The maximum absolute error of example 2 for $\boldsymbol{\delta}=\mathbf{0 . 5} \boldsymbol{\varepsilon}$

\begin{tabular}{|c|c|c|c|c|}
\hline$\varepsilon$ & $N=2^{-4}$ & $N=2^{-5}$ & $N=2^{-6}$ & $N=2^{-7}$ \\
\hline $2^{-4}$ & $5.950 \mathrm{e}-004$ & $1.56 \mathrm{e}-004$ & $3.90 \mathrm{e}-005$ & $8.00 \mathrm{e}-006$ \\
\hline $2^{-5}$ & $1.152 \mathrm{e}-003$ & $2.88 \mathrm{e}-004$ & $7.40 \mathrm{e}-005$ & $1.80 \mathrm{e}-005$ \\
\hline $2^{-6}$ & $2.233 \mathrm{e}-003$ & $5.48 \mathrm{e}-004$ & $1.43 \mathrm{e}-004$ & $3.30 \mathrm{e}-005$ \\
\hline \multicolumn{5}{|l|}{} \\
\hline Swamy et al. (2015) & $1.8632 \mathrm{e}-002$ & $9.6189 \mathrm{e}-003$ & $4.8865 \mathrm{e}-003$ & $2.4643 \mathrm{e}-003$ \\
\hline $2^{-4}$ & $2.8161 \mathrm{e}-002$ & $1.4818 \mathrm{e}-002$ & $7.6255 \mathrm{e}-003$ & $3.8713 \mathrm{e}-003$ \\
\hline $2^{-5}$ & $3.7958 \mathrm{e}-002$ & $2.0967 \mathrm{e}-002$ & $1.0977 \mathrm{e}-002$ & $5.6273 \mathrm{e}-003$ \\
\hline $2^{-6}$ &
\end{tabular}

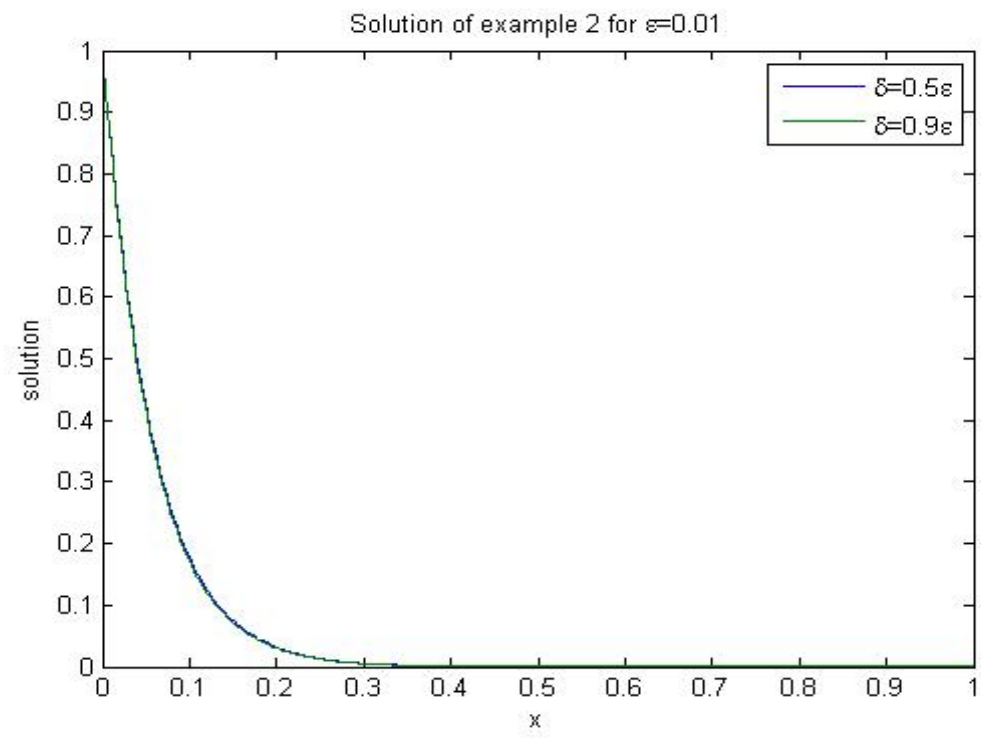

Figure 2. Numerical solution of Example 2 with different values of $\boldsymbol{\delta}$ with $\boldsymbol{\varepsilon}=0.01$

Example 3. Consider the equation obtained from equation (1) for $a(x)=0.25, b(x)=1$ with $f(x)=1$ given by

$\varepsilon y^{\prime \prime}(x)+0.25 y(x-\delta)+y(x)=1$ subject to the boundary conditions $y(x)=1,-\delta \leq$ $x \leq 0$ and $y(1)=0$.

The oscillation behavior of the obtained solution for this example is presented for four different values of $\delta$ in Figure 3. In Table 5, the maximum absolute error calculated for this example for different values of delay parameter is presented. The results are compared with those represented in literature. 
International Journal of Mathematical, Engineering and Management Sciences

Vol. 4, No. 2, 349-360, 2019

https://dx.doi.org/10.33889/IJMEMS.2019.4.2-028

Table 5. Maximum absolute error obtained for Example 3 for $\boldsymbol{\varepsilon}=\mathbf{0 . 1}$

\begin{tabular}{|c|c|c|c|}
\hline$\delta$ & $\mathrm{N}=100$ & $\mathrm{~N}=200$ & $\mathrm{~N}=400$ \\
\hline 0.03 & $1.1800 \mathrm{e}-003$ & $6.8000 \mathrm{e}-004$ & $3.0000 \mathrm{e}-004$ \\
\hline 0.05 & $1.0400 \mathrm{e}-003$ & $3.5000 \mathrm{e}-004$ & $2.8700 \mathrm{e}-003$ \\
\hline 0.09 & $1.0300 \mathrm{e}-003$ & $9.2000 \mathrm{e}-004$ & $5.9000 \mathrm{e}-004$ \\
\hline \multicolumn{5}{|l}{} \\
\hline Swamy et al. (2015) & $2.5991 \mathrm{e}-003$ & $1.2872 \mathrm{e}-003$ & $6.40390 \mathrm{e}-004$ \\
\hline 0.03 & $2.6270 \mathrm{e}-003$ & $1.3013 \mathrm{e}-003$ & $6.47505 \mathrm{e}-004$ \\
\hline 0.05 & $2.6813 \mathrm{e}-003$ & $1.3289 \mathrm{e}-003$ & $6.61390 \mathrm{e}-004$ \\
\hline
\end{tabular}
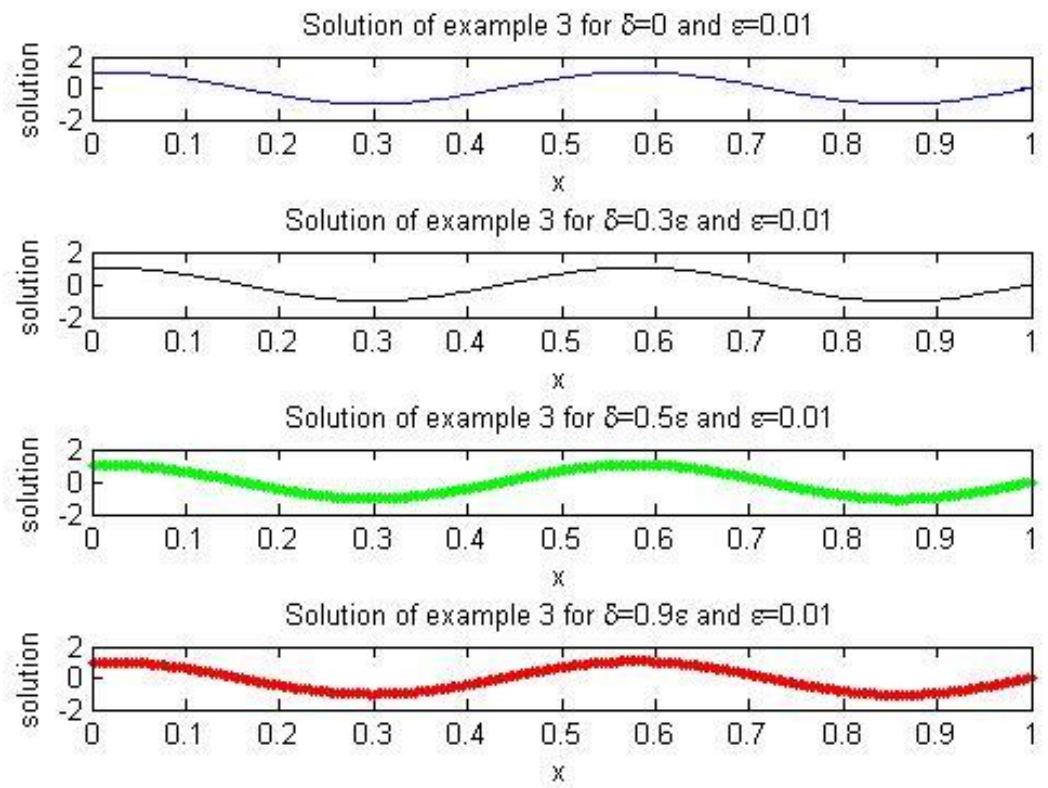

Figure 3. Numerical solution of Example 3 for different values of $\boldsymbol{\varepsilon}$ and $\boldsymbol{\delta}$

Example 4. Consider the equation $\varepsilon y^{\prime \prime}(x)+y(x-\delta)+2 y(x)=1$ to be solved with boundary conditions given by

$$
y(x)=1,-\delta \leq x \leq 0 \text { and } y(1)=0 .
$$

The solution of the considered equation with given boundary conditions shows oscillation behavior which is presented in Figure 4 for three different values of perturbation parameter and the maximum absolute error obtained for this example for different values of delay $\delta$ and $N$ is presented in Table 6. 
International Journal of Mathematical, Engineering and Management Sciences

Vol. 4, No. 2, 349-360, 2019

https://dx.doi.org/10.33889/IJMEMS.2019.4.2-028

Table 6. Maximum absolute error obtained for Example 4 for $\boldsymbol{\varepsilon}=\mathbf{0 . 1}$ and for different values of $\mathrm{N}$

\begin{tabular}{|c|c|c|c|}
\hline$\delta$ & $\mathrm{N}=100$ & $\mathrm{~N}=200$ & $\mathrm{~N}=400$ \\
\hline 0.03 & $8.8000 \mathrm{e}-004$ & $1.4000 \mathrm{e}-004$ & $2.5300 \mathrm{e}-004$ \\
\hline 0.05 & $7.6000 \mathrm{e}-004$ & $1.6000 \mathrm{e}-004$ & $3.3000 \mathrm{e}-004$ \\
\hline 0.09 & $8.3000 \mathrm{e}-004$ & $2.8000 \mathrm{e}-004$ & $2.1100 \mathrm{e}-004$ \\
\hline \multicolumn{4}{|l|}{ Swamy et al. (2015) } \\
\hline 0.03 & $1.5929 \mathrm{e}-002$ & $7.4850 \mathrm{e}-003$ & $3.6202 \mathrm{e}-003$ \\
\hline 0.05 & $1.5470 \mathrm{e}-002$ & $7.2782 \mathrm{e}-003$ & $3.5209 \mathrm{e}-003$ \\
\hline 0.09 & $2.1396 \mathrm{e}-002$ & $1.0097 \mathrm{e}-002$ & $4.8916 \mathrm{e}-003$ \\
\hline
\end{tabular}

Solution for $\varepsilon=0.01$
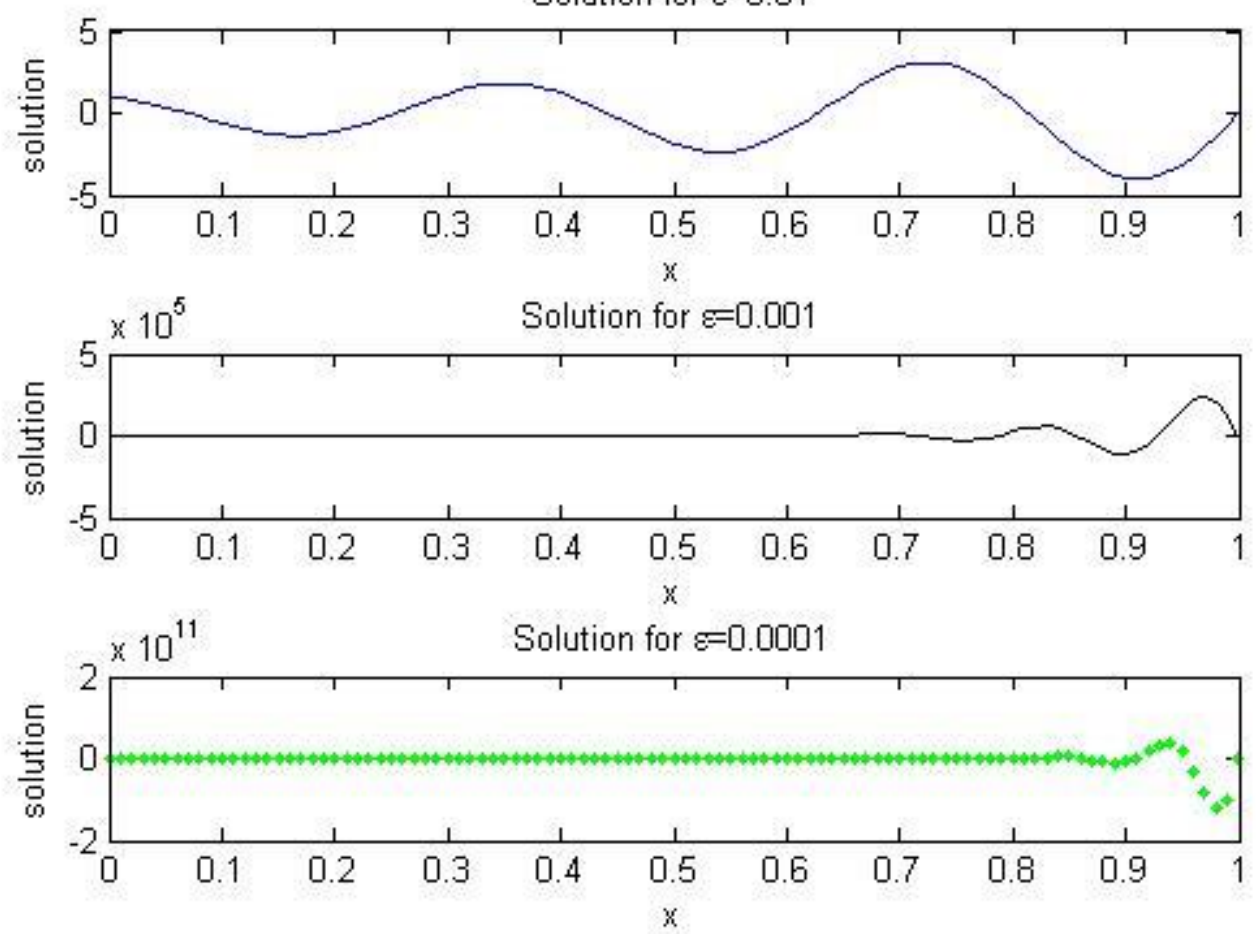

Figure 4. Solution of Example 4 for various values of $\boldsymbol{\varepsilon}$

\section{Conclusion}

A numerical approach based on trigonometric cubic B-spline function with collocation method is used to determine the solution of SPDDE. We have used uniform mesh to partition the domain. The presented scheme is applied on four numerical examples and the results obtained are shown in tables. The examples are considered to present the effect of different boundary layers. Graphs are also plotted for the solution. It is found that the results obtained by the proposed scheme are better than the results obtained by the reported method. The rate of convergence of the presented scheme is calculated as two by application of truncation error. It can be deduced that the given method is easy and efficient to apply on SPDDE. 
International Journal of Mathematical, Engineering and Management Sciences

Vol. 4, No. 2, 349-360, 2019

https://dx.doi.org/10.33889/IJMEMS.2019.4.2-028

\section{Conflict of Interest}

The authors confirm that there is no conflict of interest to declare for this publication.

\section{Acknowledgement}

The authors would like to express their sincere thanks to the referee and for their valuable suggestions towards the improvement of the paper.

\section{References}

Bhatt, N., Anand, A., Yadavalli, V. S. S., \& Kumar, V. (2017). Modeling and characterizing software vulnerabilities. International Journal of Mathematical, Engineering and Management Sciences, 2(4), 288-299.

Bocharov, G. A., \& Rihan, F. A. (2000). Numerical modelling in biosciences using delay differential equations. Journal of Computational and Applied Mathematics, 125(1-2), 183-199.

Chakravarthy, P. P., \& Kumar, K. (2017). A novel method for singularly perturbed delay differential equations of reaction-diffusion type. Differential Equations and Dynamical Systems, 1-12, DOI: 10.1007/s12591-017-0399-x.

Dag, I., Ersoy, O., \& Kaçmaz, O. (2014). The trigonometric cubic B-spline algorithm for Burgers' equation. arXiv preprint arXiv:1407.5434.

Deepika, O. S., Anand, A., \& Singh, J. N. (2017). Testing domain dependent software reliability growth models. International Journal of Mathematical, Engineering and Management Sciences, 2(3), 140149.

Derstine, M. W., Gibbs, H. M., Hopf, F. A., \& Kaplan, D. L. (1982). Bifurcation gap in a hybrid optically bistable system. Physical Review A, 26(6), 3720.

File, G., Gadisa, G., Aga, T., \& Reddy, Y. N. (2017). Numerical solution of singularly perturbed delay reaction-diffusion equations with layer or oscillatory behaviour. American Journal of Numerical Analysis, 5(1), 1-10.

Kanth, A. R., \& Kumar, P. M. M. (2018). Numerical method for a class of nonlinear singularly perturbed delay differential equations using parametric cubic spline. International Journal of Nonlinear Sciences and Numerical Simulation, 19(3-4), 357-365.

Lange, C. G., \& Miura, R. M. (1982). Singular perturbation analysis of boundary value problems for differential-difference equations. SIAM Journal on Applied Mathematics, 42(3), 502-531.

Longtin, A., \& Milton, J. G. (1988). Complex oscillations in the human pupil light reflex with "mixed" and delayed feedback. Mathematical Biosciences, 90(1-2), 183-199.

Mackey, M. C., \& Glass, L. (1977). Oscillation and chaos in physiological control systems. Science, 197(4300), 287-289.

Mahendran, R., \& Subburayan, V. (2018). Fitted finite difference method for third order singularly perturbed delay differential equations of convection diffusion type. International Journal of Computational Methods, 1840007.

Nelson, P. W., \& Perelson, A. S. (2002). Mathematical analysis of delay differential equation models of HIV-1 infection. Mathematical Biosciences, 179(1), 73-94. 
International Journal of Mathematical, Engineering and Management Sciences

Vol. 4, No. 2, 349-360, 2019

https://dx.doi.org/10.33889/IJMEMS.2019.4.2-028

Rihan, F. A. (2013). Delay differential equations in biosciences: parameter estimation and sensitivity analysis. In Recent Advances in Applied Mathematics and Computational Methods: Proceedings of the 2013 International Conference on Applied Mathematics and Computational Methods (Venice, Italy September 2013) (pp. 50-58).

Shah, N. H., Patel, S. N., Satia, M. H., \& Thakkar, F. A. (2018). Optimal control for transmission of water pollutants. International Journal of Mathematical, Engineering and Management Sciences, 3(4), 381391.

Sirisha, C. L., \& Reddy, Y. N. (2017). Numerical integration of singularly perturbed delay differential equations using exponential integrating factor. Mathematical Communications, 22(2), 251-264.

Stein, R. B. (1965). A theoretical analysis of neuronal variability. Biophysical Journal, 5(2), 173-194.

Stein, R. B. (1967). Some models of neuronal variability. Biophysical Journal, 7(1), 37-68.

Swamy, D. K., Phaneendra, K., Babu, A. B., \& Reddy, Y. N. (2015). Computational method for singularly perturbed delay differential equations with twin layers or oscillatory behaviour. Ain Shams Engineering Journal, 6(1), 391-398. 